\title{
Seroprevalencia de anticuerpos contra Toxoplasma gondii en pacientes cubanos con la infección por el VIH.
}

\section{Artículo Original}

Ivonne Martín-Hernández¹, Susana M. García-Izquierdo².

${ }^{1}$ Laboratorio de Enfermedades Infecciosas. Centro de Inmunoensayo. ${ }^{2}$ TecnoSUMA Internacional. Centro de Inmunoensayo, La Habana, Cuba.

\section{RESUMEN.}

Introducción. Con la infección del virus de inmunodeficiencia humana (VIH), el Toxoplasma gondii ha surgido como un agente patógeno oportunista importante. Esta parasitosis involucra una variedad de órganos del hospedero particularmente el tracto gastrointestinal, pulmones, miocardio, cerebro y ojos. Entre el 10-50\% de los pacientes infectados con el VIH los cuales son seropositivos para $T$. gondii desarrollan encefalitis toxoplásmica. La incidencia de este parásito en pacientes infectados con VIH depende de la existencia de toxoplasmosis latente en la población afectada.

Material y métodos. Con el uso del UMELISA Toxoplasma ${ }^{\circledR}$, fue estudiada la presencia de anticuerpos de tipo IgG a Toxoplasma gondii en 107 pacientes con VIH y 123 donantes de sangre como grupo control. La presencia de anticuerpos IgM fue determinada mediante el estuche comercial Toxonostika IgM II.

Resultados. La prevalencia de anticuerpos IgG en pacientes fue $71,96 \%$ (77/107) y en donantes $71,54 \%$
(88/123), no encontrándose diferencias significativas entre ambos grupos. Ningún caso fue seropositivo a anticuerpos IgM anti-Toxoplasma. En los pacientes con VIH/SIDA no se encontró relación entre la seroreactividad y el conteo de linfocitos CD4.

Discusión. La alta prevalencia de anticuerpos antiT. gondii en ambos grupos sugiere que esta zoonosis es endémica en Cuba. La alta morbilidad y mortalidad de la toxoplasmosis en la población infectada con VIH, justifica que la determinación y monitoreo de los anticuerpos anti-Toxoplasma sea esencial en estos pacientes. (Rev Biomed 2004; 15:215-220)

Palabras clave: VIH, SIDA, Toxoplasma gondii, Cuba.

\section{SUMMARY.}

Seroprevalence of antibodies against Toxoplasma gondii in Cuban patients with HIV infection.

Introduction. With the infection of the human

Solicitud de sobretiros: Lic. Ivonne Martín-Hernández, Centro coloborador de la OMS para el Desarrollo de enfoques genéticos en la promoción de la salud. Calle 146 No. 3102 esq. Ave. 31. Marianao, C.P. 11600, Ciudad de la Habana, Cuba.

E-mail: ivonne.martin@infomed.sld.cu

Recibido el 19/Abril/2004. Aceptado para publicación el 18/Octubre/2004.

Este artículo está disponible en http://www.uady.mx/sitios/biomedic/revbiomed/pdf/rb041543.pdf

Vol.15/No.4/Octubre-Diciembre, 2004 


\section{Martín-Hernández, SM García-Izquierdo.}

immunodeficiency virus (HIV), Toxoplasma gondii has emerged as an important opportunistic pathogenic agent. This parasitical infection involves a variety of host organs, particularly the gastrointestinal tract, lungs, myocardium, brain, and eyes. Between $10-50 \%$ of HIV-infected patients who are seropositive for $T$. gondii develop Toxoplasma encephalitis. The incidence of this parasite in HIV-infected principally patients depends on the existence of latent toxoplasmosis in the affected population.

Material and methods. UMELISA Toxoplasma®, was used to study the presence of IgG antiToxoplasma antibodies in 107 patients with HIV and 123 blood donors as a control group. The presence of $\operatorname{IgM}$ antibodies was determined using the commercial kit Toxonostika IgM II.

Results: The prevalence was 71,96\% (77/107) in patients and $71,54 \%(88 / 123)$ in blood donors, with no significant difference between the two groups. No cases were seropositive to IgM anti-Toxoplasma antibodies. No relationship between seroreactivity and CD4 T-cell counts in HIV/AIDS patients was found. Discussion. The high prevalence of $T$. gondii antibodies in both groups suggests that this zoonosis is endemic in Cuba. The high morbidity and mortality of toxoplasmosis in the HIV-infected population, justifies the routine determination and monitoring of anti-Toxoplama antibodies in these patients.

(Rev Biomed 2004; 15:215-220)

Key words: HIV, Toxoplasma gondii, IgM, IgG, Cuba.

\section{INTRODUCCIÓN.}

La toxoplasmosis es la enfermedad causada por la infección con el parásito intracelular obligado Toxoplasma gondii y constituye una infección oportunista en pacientes infectados con el virus de inmunodeficiencia humana (VIH) y con menos de 100 linfocitos T CD4/ $\mu \mathrm{L}(1,2)$. Esta parasitosis involucra una variedad de órganos del hospedero, particularmente el tracto gastrointestinal, pulmones, miocardio, cerebro y ojos. En pacientes con el síndrome de inmunodeficiencia adquirida (SIDA) se puede producir una reactivación de la infección latente con Toxoplasma gondii, cuya frecuencia depende de su prevalencia en la población general. Esta infección oportunista aparece aproximadamente en el $15 \%$ de estos pacientes y es más común en individuos que viven en el Caribe y en Francia $(3,4)$. Entre el $10-50 \%$ de los pacientes infectados con el VIH y con serología positiva para Toxoplasma desarrollan encefalitis toxoplásmica, con casi un 50\% de secuelas neurológicas y cuya mortalidad se acerca al $20 \%$. Estos porcentajes la convierten en la segunda infección oportunista más común que afecta el sistema nervioso central (SNC), en pacientes con infección por el VIH. La mayoría de los casos de toxoplasmosis en enfermos con SIDA que se han reportado, se relaciona con infección del sistema nervioso central, lo que se traduce en un deterioro de la función neurológica, la visión, la audición y pudiendo causar la muerte $(5,6)$.

La presencia de anticuerpos IgG contra este parásito se considera como un elemento diagnóstico de infección pretérita, aunque en la actualidad muchos investigadores concuerdan en que en estos pacientes los niveles de anticuerpos anti-Toxoplasma no tienen valor para el diagnóstico de esta parasitosis (7-10).

En nuestra comunidad la prevalencia de anticuerpos anti-Toxoplasma en pacientes con VIH/SIDA no ha sido reportada. Los estudios realizados de anticuerpos han sido dirigidos fundamentalmente a pacientes con sospechas clínicas de neurotoxoplasmosis (11-13). Debido al riesgo de daño al SNC y a la alta morbilidad y mortalidad de esta parasitosis en pacientes con el VIH, consideramos de importancia definir la prevalencia de anticuerpos IgM e IgG anti-Toxoplasma, en este grupo de la población cubana, contribuyendo al conocimiento de la epidemiología de la toxoplasmosis en Latinoamérica.

\section{MATERIAL Y MÉTODOS.}

En un periodo de seis meses, se colectaron un total de 107 muestras de suero de una población de pacientes infectados con el VIH (de ellos, 8 sueros pertenecientes a enfermos con SIDA), asintomáticos a toxoplasmosis, procedentes del Sanatorio para 


\section{Anticuerpos contra Toxoplasma gondii en pacientes con VIH.}

pacientes VIH/SIDA, en Santiago de las Vegas, Habana, Cuba. Como grupo control, se obtuvieron muestras de suero de 140 donantes de sangre (VIH, HbsAg y VDRL negativos), que acudieron al Banco de Sangre del Municipio Marianao de la Ciudad de la Habana.

La toma de muestra se realizó mediante punción venosa, luego se obtuvo el suero previa centrifugación. Las muestras se almacenaron a $-20^{\circ} \mathrm{C}$ hasta su posterior evaluación. Sólo se registraron los datos de edad para ambos grupos y cuenta de linfocitos CD4 para los pacientes con VIH.

El personal de salud de ambas instituciones y toda la población sometida al estudio fueron informados, obteniéndose los permisos por escrito, administrativos y éticos de las autoridades municipales y médicas. La participación fue voluntaria.

Sólo a los pacientes con VIH se les realizó la detección de anticuerpos IgM específicos. Para ello se empleó el estuche comercial Toxonostika IgM II de la casa comercial Organon Teknika SA. Para la determinación de anticuerpos IgG anti-T. gondii se emplearon los reactivos del estuche UMELISA Toxoplasma ${ }^{\circledR}$, el cual es producido en el Centro de Inmunoensayo y comercializado por la empresa TecnoSuma Internacional S.A, Cuba. Esta técnica es rápida, sencilla y confiable. Presenta una detectabilidad de $3 \mathrm{UI} / \mathrm{mL}$, los coeficientes de variación intraensayos se encuentran entre 1.4-8.0\% y los coeficientes de variación interensayos entre 4,8$10.4 \%$. Su recuperación es de 83-96\%.

El UMELISA Toxoplasma ${ }^{\circledR}$ es un ensayo inmunoenzimático heterogéneo de tipo indirecto que permite cuantificar hasta $1000 \mathrm{UI} / \mathrm{mL}$ de anticuerpos IgG anti-Toxoplasma gondii en muestras de suero, y utiliza como fase sólida tiras de poliestireno (Greiner Labortechnik), sensibilizadas previamente con un antígeno soluble de $T$. gondii (trofozoitos sonicados), suministrados por el Instituto de Medicina Tropical "Pedro Kourí". Los sueros son diluidos 1:101, y si presentan anticuerpos específicos, forman un inmunocomplejo que es reconocido posteriormente por un complejo anti-IgG humana obtenida en carnero y conjugada con fosfatasa alcalina. Entre un paso y otro, se realizaron 4 lavados de las tiras de reacción con solución reguladora de fosfato $\mathrm{pH}$ 9.8. Las incubaciones de muestras y conjugado se realizaron durante media hora a $37^{\circ} \mathrm{C}$. El volumen de reactivos utilizados en cada paso de la técnica es de $10 \mu \mathrm{L}$. Finalmente, se reveló la reacción con la adición del sustrato fluorogénico 4-metilumbeliferil fosfato y en los resultados la intensidad de la fluorescencia emitida, es proporcional a la concentración de anticuerpos presentes en la muestra. El cálculo e informe de los resultados son realizados automáticamente por el lector SUMA mediante un programa diseñado al efecto (14).

Los datos fueron almacenados y procesados por computadora, empleando el tabulador electrónico Microsoft Excel 2000. El análisis estadístico de los resultados se realizó mediante el empleo de la prueba $\mathrm{C}^{2}$ a través del programa de computación TonyStat fabricado en la Facultad de Biología de la Universidad de la Habana (15).

\section{RESULTADOS.}

No se encontró ningún paciente con posible infección reciente o reactivación, expresando en todos los casos seronegatividad a anticuerpos IgM antiToxoplasma gondii.

En este estudio se encontró una prevalencia de anticuerpos IgG anti-Toxoplasma gondii de 71,96\% (77/107) en los pacientes con VIH/SIDA y de $71,54 \%$ (88/123) en los donantes de sangre estudiados, no encontrándose diferencias significativas entre ambos grupos ( $p>0.05)$. En el cuadro 1 se comparan los resultados de seropositividad en

\section{Cuadro 1}

Prevalencia de anticuerpos IgG anti-T. gondii en pacientes con VIH y donantes de sangre en relación a grupos etarios.

\begin{tabular}{lll}
\hline $\begin{array}{l}\text { Edad } \\
\text { (años) }\end{array}$ & Pacientes & $\begin{array}{l}\text { Donantes } \\
\text { de sangre }\end{array}$ \\
\hline $15-25$ & $11 / 24(45,8 \%)$ & $18 / 38(47,4 \%)$ \\
$26-35$ & $35 / 45(77,8 \%)$ & $33 / 41(80,5 \%)$ \\
36 ó más & $31 / 38(81,6 \%)$ & $37 / 44(84,0 \%)$ \\
Total & $77 / 107(71,96 \%)$ & $88 / 123(71,54 \%)$ \\
\hline
\end{tabular}

Vol.15/No.4/Octubre-Diciembre, 2004 


\section{Martín-Hernández, SM García-Izquierdo.}

pacientes y controles en relación con la edad, apreciándose que entre pacientes y donantes, para cada grupo etario, no existen diferencias significativas ( $p>0.05)$. Puede observarse que en ambos grupos se incrementa la positividad con la edad.

La seropositividad a anticuerpos IgG antiToxoplasma encontrada en los sueros de pacientes diagnosticados con SIDA fue de 75,0\% (6/8), mientras que en los pacientes portadores del virus se encontró un $71,7 \%$ (71/99), no encontrándose diferencias estadísticas entre estos dos grupos ( $\mathrm{p}>0.05)$.

La prevalencia de anticuerpos contra $T$. gondii en los 107 pacientes con VIH fue independiente del número de linfocitos CD4, no encontrándose diferencias significativas entre los grupos con un número de linfocitos ${ }^{3} 500$ células CD4/ $\mu \mathrm{L}, 200-499$ células CD4/ $\mu \mathrm{L}, 100-200$ células CD4/ $\mu \mathrm{L}$ y £100 células CD4/ $\mu \mathrm{L},(\mathrm{p} 0,05)$ (cuadro 2).

\section{Cuadro 2}

Relación entre seropositividad de anticuerpos anti-T. gondii y asociación con número de linfocitos CD4.

\section{Células CD4/ $\mu \mathrm{L} \quad$ Prevalencia de positividad}

\begin{tabular}{|c|c|}
\hline${ }^{3} 500$ & $34 / 48(70,8 \%)$ \\
\hline 200-499 & $38 / 52(73,1 \%)$ \\
\hline $100-200$ & $3 / 4 \quad(75,0 \%)$ \\
\hline$£ 100$ & $(66,7 \%)$ \\
\hline
\end{tabular}

Total 77/107

\section{DISCUSIÓN.}

En nuestro estudio resultó interesante encontrar que las prevalencias de anticuerpos anti-T. gondii, tanto en donantes como en pacientes, fueron similares, a pesar de la disfunción de la respuesta humoral descrita en los pacientes VIH/SIDA (16). Estos resultados pueden ser explicados por los hechos siguientes: 1) En el 96,1\% de los casos (74/77) se encontraron títulos bajos entre 12,5 y $125 \mathrm{UI} / \mathrm{mL}$ y el $3,9 \%$ restante entre 126-200 UI/mL, pudiendo inferir que el contacto con el parásito ocurrió de manera esporádica en el pasado. 2) En Cuba los porcentajes de seroreactividad a $T$. gondii en población general oscilan entre el $50 \%$ y $75 \%$ (17-24), por lo que no resulta extraño encontrar una alta prevalencia aún en este tipo de paciente.

Internacionalmente, la seropositividad a Toxoplasma en pacientes infectados con el VIH varía mucho, comportándose en países como Estados Unidos entre el 10-45\% y en Europa y Africa entre el 50-78\% (3, 7-8, 25-27). En Malasia, Tailandia y México se han encontrado un 21\%, 23,2\% y $50 \%$, respectivamente (28-30). En Brasil, en los pacientes que mueren por SIDA, el principal patógeno que daña el SNC es el Toxoplasma gondii.(31). En pacientes cubanos con VIH/SIDA, con síntomas de neurotoxoplasmosis, se ha reportado hasta un $92 \%$ de seropositividad a $T$. gondii, constituyendo en algunos estudios la causa mayor de muerte en estos enfermos (11-13).

A diferencia de otros reportes encontrados en la literatura $(3,32)$, obtuvimos como resultado de nuestro estudio, similares seroprevalencias de anticuerpos anti-Toxoplasma en los enfermos de SIDA y en los pacientes portadores del VIH.

Al igual que otros autores no encontramos asociación entre presencia de anticuerpos y número celular de linfocitos CD4, considerado como un posible factor de riesgo de la toxoplasmosis encefálica (4).

En los pacientes con infección por VIH, la toxoplasmosis puede ocurrir con bajos niveles de anticuerpos. Raramente están presentes las IgM específicas y con la disminución del número de linfocitos CD4, se incrementa el riesgo de padecer la infección, siendo mayor en pacientes con menos de 100 células CD4/ $\mu \mathrm{L}(6,16,33,34)$. Atendiendo a estos hechos, a los dos casos que se encontraron con menos de 100 células CD4/ $\mu \mathrm{L}$, se les realizó tomografía axial computarizada de cráneo y una exhaustiva evaluación oftalmológica. Los resultados de estos estudios no reflejaron ningún daño en estos individuos, pero se recomendó el seguimiento y profilaxis en los mismos.

La alta prevalencia de anticuerpos anti-T. gondii obtenida en nuestro estudio, para ambos grupos poblacionales, indica una vez más que esta zoonosis

\section{Revista Biomédica}




\section{Anticuerpos contra Toxoplasma gondii en pacientes con VIH.}

es endémica en Cuba. La alta proporción de reactivación de esta parasitosis en los pacientes inmunocomprometidos justifica que la determinación rutinaria de anticuerpos anti-T. gondii, quede incluida en los protocolos de diagnóstico y en el establecimiento de tratamiento profiláctico en los pacientes con VIH/SIDA, ya que un porcentaje alto de ellos estaría en riesgo de desarrollar toxoplasmosis encefálica.

\section{REFERENCIAS.}

1.- Wong SY, Remington JS. Biology of Toxoplasma gondii. AIDS 1993; 7: 299-318.

2.- Okome M, Mbounja ME, Kombila M. Spectrum of opportunistic infections in subjects infected with HIV. Sante 2000; 10: 329-37.

3.- Maiga I, Kiemtore P, Tounkara A. Prevalence of antiToxoplasma in patients with acquired immunodeficiency syndrome and blood donors in Bamako. Bull Soc Pathol Exot 2001; 94: 268-70.

4.- Góngora RA, Gónzalez P, Castro C, Alvarez R, Pavia N, Lara D, et al. Anticuerpos contra Toxoplasma gondii en pacientes con VIH en Yucatán. Rev Invest Clin 1998; 50: 419-22.

5.- Luft BJ, Hafner R, Korzun AH. Toxoplasmic encephalitis in patients with adquired immunodeficiency syndrome. N Engl J Med 1993; 329: 995-1000.

6.- Kasper LH. Toxoplasma infection. In: Braunwald E, Fauci AS, Kasper DL, Hauser SL, Longo DL, Jameson JL, eds. Harrinson's Principles of Internal Medicine. 15 th ed. New York: McGraw-Hill 2001.p. 1222-7.

7.- Grant HI, Gold JW. Toxoplasma gondii serology in HIVinfected patients, the development of central nervous system toxoplasmosis in AIDS. AIDS 1990; 6: 519-21.

8.- Holliman RE. Serological study of the prevalence of toxoplamosis in asympotamatic patients infected with human immunodeficiency virus. Epidemiol Infect 1990; 105:415-8.

9.- Hayde M, Salzer HR, Gittler G, Aspock H, Pollak A. Microparticle enzyme immunoassay (MEIA) for Toxoplasma specific immunoglobulin $\mathrm{G}$ in comparison to the SabinFeldman dye test. A pilot study. Wien Klin Wochenschr 1995; 107:133-6
10.- Pinon JM, Foudrinier F, Mougeot G, Marx C, Aubert D, Toupance $\mathrm{O}$, et al. Evaluation of risk and diagnostic value of quantitative assays for anti-Toxoplasma gondii immunoglobulin A ( $\operatorname{Ig} \mathrm{A}), \operatorname{IgE}$ and $\operatorname{IgM}$ and analytical study of specific IgG in immunodefient patients. J Clin Microbiol 1995; 33: 878-84.

11.- Rodríguez MS, Fernández T, Ginorio D, Capó V, Garcia G, Cox R. Contribución al conocimiento de la encefalitis por Toxoplasma en pacientes VIH/SIDA 1998-2001. II Foro en VIH / SIDA / ITS de América Latina y el Caribe. Cuba, Abril 2003. CD-ROOM Memorias Foro 2003.

12.- Medina E. Características clínicas de la infección por el VIH-SIDA en la provincia Holguín, Cuba. Años 1986 al 2000. II Foro en VIH / SIDA / ITS de América Latina y el Caribe. Cuba, Abril 2003. CD-ROOM Memorias Foro 2003.

13.- Véliz DM. Mortalidad de la epidemia del VIH/SIDA en Pinar del Río 1991-2002. II Foro en VIH / SIDA / ITS de América Latina y el Caribe. Cuba, Abril 2003. CD-ROOM Memorias Foro 2003.

14.- Fernández JL. Screening Technology Development. The Cuban experience. $8^{\text {th }}$ International Symposium on Neonatal Screening and Inaural Meeting of International Society for Neonatal Screening. Sidney, Australia: Abstract Book; 1991.

15.- Sigarroa A. Manual de clases prácticas de biometría y diseño experimental. 1a ed. Cuba: Editora Pueblo y Educación; 1987.

16.- Góngora-Biachi RA, Gónzalez-Martínez P, Puerto F, Castro-Sansores C. Inmunopatogénesis de la infección por el virus de Inmunodeficiencia humana. Rev Biomed 1991; 2: 207-16.

17.- Acosta C, Pérez X, García R. Presencia de anticuerpos IgG anti-Toxoplasma gondii en embarazadas residentes en Ciudad de la Habana. Rev Biomed 2001; 12: 250-54.

18.- González T, Bacallao J, García C, Molina JR. Prevalencia de anticuerpos anti-Toxoplasma gondii en una población de mujeres embarazadas en Cuba. Gac Méd Méx 1991; 131 : 499-03.

19.- Collazo JE, López R, Ginorio D, Llamos R, Contreras R. Anticuerpos IgG anti-Toxoplasma gondii en pacientes con síntomas atribuíbles a toxoplasmosis. Biomed 1993; 13: 17982.

20.- Martinez R, Bacallao R, Alberti E, Alfonso I. Prevalence of toxoplasmosis in pregnant women of the province of $\mathrm{La}$ 


\section{Martín-Hernández, SM García-Izquierdo.}

Habana. Rev Inst Trop Sao Paulo 1994; 36: 445-50.

21.- Thulliez Ph, Derouin F, Peyron F, Wallon M, Candolfi E. Toxoplasmosis and diagnostic. 1a ed. Madrid: Coimpress SA; 1996. p. 11.

22.- Sánchez A, Martín I, García S. Estudio de reactividad a Toxoplasma gondii en embarazadas de las provincias Ciudad de la Habana y Pinar del Río, Cuba. Bioquimia 2003; 111: 3-8.

23.- López R, Fano R, Contreras R, Font L. Anticuerpos IgG anti- Toxoplasma gondii en cubanos donantes de sangre. Rev Lat Am Microbiol 1993; 35: 207-10.

24.- Martín I, García SM. Prevalencia de anticuerpos IgG contra Toxoplasma gondii en donantes de sangre cubanos. Rev Biomed 2003; 14: 247-51.

25.- Pauwels A, Meyohas MC, Eliaszewicz M, Legendre C, Mougeot G, Frottier J, et al. Toxoplasma colitis in the acquired imunodeficiency syndrome. Am J Gastroenterol 1992; 87: $518-9$.

26.- Porter SB, Sande MA. Toxoplasmosis of the central nervous system in the acquired immunodeficiency syndrome. N Engl J Med 1993; 327: 1643-8.

27.- Reparaz PJ, Regalados DCJ, Urix Y, Ayestran J. Lesiones cerebrales pacientes con sindrome de inmunodeficiencia adqurida. Enferm Infec Microbiol 1991; 9: 85-9.

28.- Nissapatorn V, Kamarulzaman A, Init I, Tan LH, Rohela M, Norliza A, et al. Seroepidemiology of toxoplasmosis among HIV-infected patients and healthy blood donors. Med J Malaysia 2002; 57: 304-10.

29.- Sukthana Y, Chintana T, Lekkla A. Toxoplasma gondii antibody in HIV-infected persons. J Med Assoc Thai 2000; 83: 681-4.

30.- Galvan ML, Valdez V, Vargas G, Jimenez O, Garcia C, Vielma M. Prevalence of IgG and IgM anti-Toxoplasma antibodies in patients with HIV and acquired immunodeficiency syndrome (AIDS). Rev Soc Bras Med Trop 1997; 30: 465-7.

31.- Zufferey J, Sugar A, Rudaz P, Bille J, Glauser MP, Chave JP. Prevalence of latent toxoplasmosis and serological diagnosis of active in HIV-positive patients. Eur J Clin Microbiol 1993;12:591-5.

32.- Wainstein MU, Ferreira L, Wolfenbuttel L, Golbspan L, Sprint E, Kronfeld M, et al. Achados neuropatológicos na síndrome da imunodeficiência adquirida (SIDA). Revisão de 138 casos. Rev Soc Bras Med Trop 1992; 25: 95-9.

33.- Stepick-Biek P, Thulliez P, Araujo FG, Remington JS. IgA antibodies for the diagnosis of congenital and acquired toxoplasmosis. J Med Microbiol 1990; 32: 25-31.

34.- Holliman RE. Toxoplasmosis and the acquired immune deficiency syndrome. J Infect Dis 1988; 16: 121-8.

\section{Revista Biomédica}

